\title{
Nonparametric Estimation in a Stochastic Volatility Model
}

\author{
Jürgen Franke* Wolfgang Härdle ${ }^{\dagger} \quad$ Jens-Peter Kreiss ${ }^{\ddagger}$ \\ March 31, 1998
}

\begin{abstract}
In this paper we derive nonparametric stochastic volatility models in discrete time. These models generalize parametric autoregressive random variance models, which have been applied quite successfully to financial time series. For the proposed models we investigate nonparametric kernel smoothers. It is seen that so-called nonparametric deconvolution estimators could be applied in this situation and that consistency results known for nonparametric errorsin-variables models carry over to the situation considered herein.
\end{abstract}

\section{Introduction}

Many methods of financial engineering like option pricing or portfolio management crucially depend on the stochastic model of the underlying asset. If $S(t)$ denotes the stock price at time $t$, then, e.g., the Black-Scholes approach to option pricing is based on modelling $\log S(t)$ as a Wiener process with drift $\mu$ and diffusion coefficient or volatility $\sigma$ :

$$
d(\log S(t))=\alpha d t+\sigma d W(t)
$$

where $W(t)$ is a standard Wiener process. This particular model is known to be inappropriate in various circumstances. For instance, $\sigma$ can no longer be assumed to be constant if the time up to exercising the option is rather short. Replacing the constant $\sigma$ by a positive stochastic process $\sigma(t)$ we arrive at the following equation for the asset price:

$$
d(\log S(t))=\alpha d t+\sigma(t) d W(t) .
$$

In the literature, several specific parametric models for the stochastic volatility $\sigma(t)$ have been proposed and used for option pricing. Here, we restrict ourselves to models

\footnotetext{
*Universität Kaiserslautern, Erwin-Schrödinger-Straße, D-67663 Kaiserslautern, Germany

${ }^{\dagger}$ Humboldt-Universität zu Berlin, Spandauer Straße 1, D-10178 Berlin, Germany

†Technische Universität Braunschweig, Pockelsstraße 14, D-38106 Braunschweig, Germany
} 
which characterize $\sigma(t)$ as the solution of a stochastic differential equation for $\log \sigma(t)$ known up to a few parameters. An example is the equation

$$
d(\log \sigma(t))=\lambda(\kappa-\log \sigma(t)) d t+\gamma d W^{*}(t)
$$

considered by Scott (1987, 1991), Wiggins (1987) and Chesney and Scott (1989). Here, $W^{*}(t)$ is another standard Wiener process correlated with $W(t)$ of $(1.1)$

$$
d W(t) d W^{*}(t)=\rho d t
$$

and $\alpha$ of (1.1), $\lambda, \kappa, \gamma$ and $\rho$ are the unknown model parameters. Other models of a similar structure have been proposed in the literature.

To help to answer the question which stochastic volatility model is appropriate for a particular data set we consider a rather general type of model avoiding the assumption of a particular parametric form of the equation defining $\sigma(t)$. At the beginning, we discretize time, as is also frequently done for parametric models for the purpose of estimating the model parameters. The log-volatility will then satisfy a general nonlinear stochastic difference equation or nonlinear autoregressive scheme. As $\sigma(t)$ is not directly observable, the now quite familiar kernel estimates for the autoregression function are not applicable. We use instead nonparametric deconvolution estimators similar to those discussed in regression analysis by Fan and Truong (1993). These estimators are consistent and provide a convenient tool for exploratory data analysis helping in the decision which particular parametric model to choose for further analysis of the data.

\section{A nonparametric stochastic volatility model}

We consider some asset with price $S(t)$ at time $t$ and, following Taylor (1994), define the return from an integer time $t-1$ to time $t$ as

$$
R_{t}=\log \frac{S(t)}{S(t-1)}
$$

To estimate a stochastic volatility model like (1.1) and (1.2), discretized versions of these equations are considered. Wiggins (1987) and Chesney and Scott (1989) use the Euler approximation

$$
\begin{gathered}
R_{t}=\mu+\sigma_{t-1} W_{t} \\
\log \sigma_{t}=\alpha+\phi\left\{\log \sigma_{t-1}-\alpha\right\}+\vartheta W_{t}^{*}
\end{gathered}
$$

$\left(W_{t}, W_{t}^{*}\right)$ denote i.i.d. bivariate standard normal random variables with zero mean and correlation $\rho$. In (2.1), the lagged quantity $\sigma_{t-1}$ appears as the stochastic volatility for period $t$. This is rather advantageous for statistical purposes, as we will 
clearly see later on.

As another simplification of (1.1), Taylor (1994) considers

$$
R_{t}=\mu+\sigma_{t} W_{t},
$$

and he called (2.1), (2.2) a lagged autoregressive random variance (LARV) model, as $\log \sigma_{t}$ follows a linear autoregressive scheme. Analogously, (2.3), (2.2), together, is called a contemporaneous autoregressive random variance (CARV) model.

In this paper, we consider nonparametric generalizations of these models. We start with the lagged case and study it in detail, whereas we give a short discussion of the contemporaneous case at the end of Section 3.

We replace (2.2) by a nonlinear nonparametric model for $\xi_{t}=\log \sigma_{t}$ :

$$
\xi_{t}=m\left(\xi_{t-1}\right)+\eta_{t},
$$

where $\eta_{t}$ denote i.i.d. zero-mean normal random variables with variance $\sigma_{n}^{2}$, and $m$ is an arbitrary autoregression function for which we only require certain smoothness assumptions.

In order to ensure that the Markov chain $\left(\xi_{t}\right)$ possesses nice probabilistic properties - e.g. geometric ergodicity and $\beta$-mixing (absolute regularity) or $\alpha$-mixing (strongly mixing) with geometrically decaying mixing coefficients - it suffices (because of the assumption of normally distributed innovations $\eta_{t}$ ) to assume an appropriate drift condition on $m$, e.g.

$$
\limsup _{|x| \rightarrow \infty}\left|\frac{m(x)}{x}\right|<1,
$$

cf. Doukhan (1994), Proposition 6 (page 107). Then, in particular, $\xi_{t}$ has a unique stationary distribution with density $p_{\xi}$.

We want to estimate $m$ using kernel-type estimates. The usual Nadaraya-Watson estimates are, however, not applicable as we cannot observe the volatility $\sigma_{t}$ or its logarithm $\xi_{t}$ directly. The available data are the asset prices $S_{t}$ or the returns $R_{t}$ which are related to $\sigma_{t}$ by $(2.1)$. Taking logarithms and using the abbreviations

$$
X_{t}=\frac{1}{2} \log \left(R_{t}-\mu\right)^{2}-\mu_{\varepsilon}, \quad \varepsilon_{t}=\frac{1}{2} \log W_{t}^{2}-\mu_{\varepsilon}
$$

with $\mu_{\varepsilon}=\mathcal{E}\left(\frac{1}{2} \log W_{t}^{2}\right)=-0.63518 \ldots(\operatorname{Scott}(1987))$, we get

$$
X_{t}=\xi_{t-1}+\varepsilon_{t},
$$

where the $\varepsilon_{t}$ are i.i.d. zero-mean random variables distributed as $\frac{1}{2}$ times the $\log$ arithm of a $\chi_{1}^{2}$-random variable centered around 0 . The correlation between the 
standard normal random variable $W_{t}$, appearing in the definition of $\varepsilon_{t}$, and $\eta_{t}$ of (2.4) is $\rho .(2.4),(2.5)$, together, form a nonparametric autoregressive model with errors-in-variables as $\xi_{t}$ cannot be observed directly but is known only through its convolution with the i.i.d. random variables $\varepsilon_{t}$. Plugging (2.5) into (2.4) we obtain the following equation for $X_{t}$ alone

$$
X_{t}=m\left(X_{t-1}-\varepsilon_{t-1}\right)+\eta_{t-1}+\varepsilon_{t} .
$$

Remark Assumption (A.1) also implies geometric ergodicity including geometrically $\beta$ - and strong mixing for the process $\left(X_{t}\right)$.

\section{Kernel estimates for the autoregressive volatil- ity function}

Fan and Truong (1993) have studied a nonparametric regression model with errorsin-variables similar to the nonparametric autoregressive model (2.4), (2.5). Following their approach, we construct nonparametric estimates for $m$ based on a sample $X_{1}, \ldots, X_{T}$. Let us assume that the parameter $\mu$, which is the expectation of the returns $R_{t}$, is known such that the $X_{t}$ are observable. From applications it can be justified that this expectation is close to zero. In case $\mu \neq 0$, the returns have to be centered before the procedure described below should be applied.

If we could observe $\xi_{1}, \ldots, \xi_{T}$ then we could estimate their stationary density, $p_{\xi}(x)$ by the kernel estimate

$$
\hat{p}_{\xi}(x, h)=\frac{1}{T h} \sum_{t=1}^{T} K\left(\frac{x-\xi_{t}}{h}\right),
$$

where $K$ denotes a probability density and $h>0$ denotes the bandwidth. The strongly mixing property of $\left(\xi_{t}\right)$, which is ensured by (A1), immediately implies consistency via a covariance inequality.

As we only observe $X_{1}, \ldots, X_{T}$, whose stationary density is the convolution of $p_{\xi}$ with the known density of the i.i.d. random variables $\varepsilon_{t}$, we have to use a deconvolution density estimate instead:

$$
\hat{p}(x, h)=\frac{1}{2 \pi} \int_{-\infty}^{\infty} e^{-i w x} \phi_{K}(w h) \frac{\hat{\phi}_{x}(w)}{\phi_{\varepsilon}(w)} d w
$$

with

$$
\begin{aligned}
& \phi_{\varepsilon}(w)=\mathcal{E} e^{i w \varepsilon_{1}}, \text { the characteristic function of } \varepsilon_{t}, \\
& \phi_{K}(w)=\int_{-\infty}^{\infty} e^{i w x} K(x) d x, \text { the Fourier transform of the kernel } K, \\
& \hat{\phi}_{x}(w)=\frac{1}{T} \sum_{t=1}^{T} e^{i w X_{t}}, \text { the sample characteristic function of } X_{1}, \ldots, X_{T} .
\end{aligned}
$$

The bandwidth $h$, depending on the sample size $T$, acts as a smoothing parameter as usual. For i.i.d. observations $\xi_{1}, \ldots, \xi_{T}$, the estimate $\hat{p}(x, h)$ for $p_{\xi}(x)$ has been 
investigated in detail by Stefanski and Carroll (1990), Carroll and Hall (1988), Fan (1991a,b) and Liu and Taylor (1989). Note that (3.1) can be written as a kernel estimator similar to $\hat{p}_{\xi}(x, h)$, namely

$$
\hat{p}(x, h)=\frac{1}{T h} \sum_{t=1}^{T} K_{h}\left(\frac{x-X_{t}}{h}\right)
$$

with a kernel $K_{h}$ depending on $h$ and on the known distribution of the $\varepsilon_{t}$

$$
K_{h}(x)=\frac{1}{2 \pi} \int_{-\infty}^{\infty} e^{-i w x} \frac{\phi_{K}(w)}{\phi_{\varepsilon}(w / h)} d w
$$

Remark. It should be noted, that without knowing anything of the distribution of the $\varepsilon_{t}$ it is completely impossible to recover the stationary density $p_{\xi}$.

Now, the nonparametric estimate for $m(x)$ is defined as a Nadaraya-Watson estimate with kernel $K_{h}$ and with $X_{t}$ replacing $\xi_{t}$, more exactly

$$
\hat{m}(x, h)=\frac{1}{T h} \cdot \sum_{t=1}^{T} K_{h}\left(\frac{x-X_{t}}{h}\right) X_{t+1} / \hat{p}(x, h) .
$$

In order to apply this estimator it is necessary to evaluate the characteristic function $\phi_{\varepsilon}$ of $\varepsilon_{t}$ and to make use of a kernel $K$ for which the Fourier transform $\phi_{K}$ takes a convenient form. Concerning the explicit form and the asymptotic behaviour of $\phi_{\varepsilon}$ we have the following result.

Lemma 3.1: Assume $W \sim \mathcal{N}(0,1)$, and let the density of the standard normal distribution be $\varphi$. The distribution of the centered random variable $\varepsilon=\frac{1}{2} \log W^{2}-\mu_{\varepsilon}$ possesses the following density

$$
p_{\varepsilon}(x)=2 \varphi\left(e^{x+\mu_{\varepsilon}}\right) e^{x+\mu_{\varepsilon}}, \quad x \in \mathbb{R} .
$$

Here $\mu_{\varepsilon}=(\kappa+\log 2) / 2 \approx 0.63518$ ( $\kappa$ denotes Eulers constant).

Let us denote by $\Gamma$ the Gamma function. We have

$$
\phi_{\varepsilon}(w)=\frac{e^{\left(\frac{\log 2}{2}-\mu_{\varepsilon}\right) i w}}{\sqrt{\pi}} \Gamma\left(\frac{1+i w}{2}\right), w \in \mathbb{R} .
$$

Concerning the tail behaviour of $\phi_{\varepsilon}$ we have for all $d_{0}, d_{1}$ with $0<d_{0}<\sqrt{2}<d_{1}<$ $\infty$ :

$$
d_{0} e^{-|w| \pi / 4} \leq\left|\phi_{\varepsilon}(w)\right| \leq d_{1} e^{-|w| \pi / 4} \text { as }|w| \longrightarrow \infty
$$


Proof: The explicit expressions for $p_{\varepsilon}$ and $\phi_{\varepsilon}$ can be obtained by direct computation, while (3.4) is an immediate consequence of the tail-behaviour of $\Gamma$, which can be found for example in Gradstein and Ryshik (1981) (No. 8.328, page 331).

Now, let us investigate the asymptotic behaviour of the kernel estimator $\hat{m}(\cdot, h)$, cf. (3.3). We have

$$
\hat{m}(x, h)-m(x)=\frac{\frac{1}{T h} \sum_{t} K_{h}\left(\frac{x-X_{t}}{h}\right)\left(X_{t+1}-m(x)\right)}{\frac{1}{T h} \sum_{t} K_{h}\left(\frac{x-X_{t}}{h}\right)} .
$$

The following lemmas imply the consistency of $\hat{m}(\cdot, h)$.

Lemma 3.2: Assume that $m$ is twice continuously differentiable and that $p_{\xi}$ is continuously differentiable. Assume that $\phi_{K}$ has a bounded support, $\left[-M_{0}, M_{0}\right]$ say, and that $h=h(T)=c / \log T$ where $c>M_{0} \pi / 2$.

$$
\begin{aligned}
E \frac{1}{T h} \sum_{t} K_{h}\left(\frac{x-X_{t}}{h}\right)\left(X_{t+1}-m(x)\right) & =\int_{-\infty}^{\infty}\{m(u)-m(x)\} \frac{1}{h} K\left(\frac{x-u}{h}\right) p_{\xi}(u) d u \\
& =O\left(h^{2}\right) \\
\text { (ii) } \operatorname{Var}\left(\frac{1}{T h} \sum_{t} K_{h}\left(\frac{x-X_{t}}{h}\right)\left(X_{t+1}-m(x)\right)\right) & =o(1) .
\end{aligned}
$$

Lemma 3.3: Assume that $p_{\xi}$ is twice times continuously differentiable. Assume that $\phi_{K}$ has a bounded support, $\left[-M_{0}, M_{0}\right]$, say, and that $h=h(T) \sim c / \log T$ where $c>M_{0} \pi / 2$. Then

$$
\begin{aligned}
E \frac{1}{T h} \sum_{t} K_{h}\left(\frac{x-X_{t}}{h}\right) & =\int_{-\infty}^{\infty} p_{\xi}(u) \frac{1}{h} K\left(\frac{x-u}{h}\right) d u \\
& =p_{\xi}(x)+O\left(h^{2}\right)
\end{aligned}
$$

(ii) $\operatorname{Var}\left(\frac{1}{T h} \sum_{t} K_{h}\left(\frac{x-X_{t}}{h}\right)\right)=o(1)$.

As an immediate consequence of Lemma 3.2 and 3.3 we obtain

Theorem 3.4: Under the assumptions of Lemma 3.2 and 3.3 we obtain for all $x \in \mathbb{R}$

$$
(\log T)^{2}(\hat{m}(x, h)-m(x))=O_{p}(1) .
$$

The nonparametric generalization of the contemporaneous autoregressive random variance model, where

$$
X_{t}=\xi_{t}+\varepsilon_{t}
$$


holds instead of (2.5), while the structure of $\left(\xi_{t}\right)$ stated in (2.4) remains valid, is much more complicated to deal with. The problems arise from the fact that $\xi_{t}$ and $\varepsilon_{t}$ are not independent (as $\xi_{t-1}$ and $\varepsilon_{t}$ were before). To see this recall that $\xi_{t}$ depends on $\eta_{t}$ which itself is correlated to $W_{t}$ (correlation $\rho$ ) appearing in the definition of $\varepsilon_{t}$. Thus, the stationary density of our observations $X_{t}$ is for the contemporaneous case not the convolution of $p_{\xi}$ (which we are interested in) with the known density of the i.i.d. random variables $\varepsilon_{t}$. To overcome the difficulties one could assume that $\rho=0$ which together with the assumption of normality for the distribution of $(\eta, W)$ implies independence even of $\varepsilon_{t}$ and $\xi_{t}$. Under this assumption $\rho=0$ all above results remain valid as can be easily seen.

In case we want to stay with the assumption $\rho \neq 0$ one has to look for another possibility to estimate $p_{\xi}$. One proposal may be as follows. Since

$$
X_{t}=\xi_{t}+\varepsilon_{t}=m\left(\xi_{t-1}\right)+\left(\eta_{t}+\varepsilon_{t}\right)
$$

we could estimate the characteristic function of $\mathcal{L}\left(m\left(\xi_{0}\right)\right)$ by $\hat{\phi}_{x}(w) / \phi_{\eta+\varepsilon}(w)$. Here $\phi_{n+\varepsilon}$ denotes the characteristic function of the known distribution of $\eta_{1}+\varepsilon_{1}$. Now $\xi_{1}=m\left(\xi_{0}\right)+\eta_{1}$ which suggests the following deconvolution estimator for $p_{\xi}$

$$
\begin{aligned}
\tilde{p}(x, h) & =\frac{1}{2 \pi} \int_{-\infty}^{\infty} e^{-i w x} \phi_{K}(w h) \frac{\hat{\phi}_{x}(w)}{\phi_{\eta+\varepsilon}(w)} \phi_{\eta}(w) d w \\
& =\frac{1}{T h} \sum_{t=1}^{T} \tilde{K}_{h}\left(\frac{x-X_{t}}{h}\right)
\end{aligned}
$$

where $\tilde{K}_{h}(n)=\frac{1}{2 \pi} \int_{-\infty}^{\infty} e^{-i w n} \phi_{K}(w) \frac{\phi_{\eta}(w / h)}{\phi_{\eta+\varepsilon}(w / h)} d w$.

Finally, as a nonparametric estimator for $m$ we propose

$$
\tilde{m}(x, h)=\frac{1}{T h} \sum_{t=1}^{T} \tilde{K}_{h}\left(\frac{x-X_{t}}{h}\right) X_{t+1} / \tilde{p}(x, h) .
$$

We have, as before

Lemma 3.5: Under suitable assumptions we have

$$
E \tilde{p}(x, h)=\int_{\mathbb{R}} p_{\xi}(x-h u) K(u) d u=p_{\xi}(x)+O\left(h^{2}\right) .
$$

In order to obtain consistency of $\hat{p}(x, h)$ we computed above the variance and obtained that it converges to zero. For the proof (cf. proof of Lemma 3.3) it was rather essential to know the asymptotic behaviour of the characteristic function $\phi_{\varepsilon}$ appearing in the denominator of $K_{h}$. Similarily, we need for a consistency result for $\tilde{p}(x, h)$ some information on the asymptotic behaviour of $\phi_{\varepsilon+\eta}$, which seems to be a rather 
delicate problem. A direct computation of $\phi_{\varepsilon+\eta}(w)$ leads to explicit expressions containing functions related to the so-called parabolic-cylinder functions $D_{\nu}(x)$. The argument $w$ appears in the argument and in the parameter of $D$, and we were not able to quantify the asymptotic behaviour of such functions as $|w| \longrightarrow \infty$.

The same problems arise when dealing with the numerator of $\tilde{m}(x, h)$, For the numerator even the computation of its expectations does not lead to such nice expressions as in the lagged case.

\section{Proofs.}

\section{Proof of Lemma 3.2:}

(i) The expectation is equal to

$$
\begin{aligned}
& \frac{1}{h} E K_{h}\left(\frac{x-X_{1}}{h}\right)\left(X_{2}-m(x)\right) \\
= & \frac{1}{h} E K_{h}\left(\frac{x-\xi_{0}-\varepsilon_{1}}{h}\right)\left(m\left(\xi_{0}\right)+\eta_{1}+\varepsilon_{2}-m(x)\right) \\
= & \frac{1}{h} E K_{h}\left(\frac{x-\xi_{0}-\varepsilon_{1}}{h}\right)\left(m\left(\xi_{0}\right)-m(x)\right)+\frac{1}{h} E K_{h}\left(\frac{x-\xi_{0}-\varepsilon_{1}}{h}\right) \eta_{1} .
\end{aligned}
$$

Recall that $E \varepsilon_{2}=0$ and that $\varepsilon_{2}$ is independent of $\xi_{0}$ and $\varepsilon_{1}$. Unfortunately $\eta_{1}$ and $\varepsilon_{1}$ are not independent. But, because of the independence of $\xi_{0}$ and $\left(\varepsilon_{1}, \eta_{1}\right)=\left(\frac{1}{2} \log W_{1}^{2}-\mu_{\varepsilon}, \eta_{1}\right)$ and $W_{1} \sim \mathcal{N}(0,1)$

$$
\begin{aligned}
& E K_{h}\left(\frac{x-\xi_{0}-\varepsilon_{1}}{h}\right) \eta_{1} \\
= & \int_{\mathbb{R}^{3}} K_{h}\left(\frac{x-u-\frac{1}{2} \log w^{2}+\mu_{\varepsilon}}{h}\right) v p_{\xi}(u) p_{\eta \mid W=w}(v) \varphi(w) d u d v d w \\
= & \int_{\mathbb{R}^{2}} K_{h}\left(\frac{x-u-\frac{1}{2} \log w^{2}+\mu_{\varepsilon}}{h}\right) \rho \sigma_{\eta} w p_{\xi}(u) \varphi(w) d u d w
\end{aligned}
$$

since the conditional distribution of $\eta$ given $W=w$ is $\mathcal{N}\left(\rho \sigma_{\eta} w, \sigma_{\eta}^{2}\left(1-\rho^{2}\right)\right)$ by our assumptions. The latter integral is equal to zero by symmetry arguments (recall that the normal density $\varphi$ is a symmetric function). Thus, the expectation under investigations equals

$$
\begin{aligned}
& \frac{1}{h} E K_{h}\left(\frac{x-\xi_{0}-\varepsilon_{1}}{h}\right)\left(m\left(\xi_{0}\right)-m(x)\right) \\
= & \frac{1}{h} \int_{\mathbb{R}^{2}} K_{h}\left(\frac{x-u-v}{h}\right)(m(u)-m(x)) p_{\xi}(u) p_{\varepsilon}(v) d u d v \\
= & \frac{1}{2 \pi h} \int_{\mathbb{R}^{3}} e^{-i \frac{w}{h}(x-u-v)} \frac{\phi_{K}(w)}{\phi_{\varepsilon}\left(\frac{w}{h}\right)}(m(u)-m(x)) p_{\xi}(u) p_{\varepsilon}(v) d u d v d w \\
= & \frac{1}{2 \pi h} \int_{\mathbb{R}^{2}} e^{-i \frac{w}{h}(x-u)} \phi_{K}(w)(m(u)-m(x)) p_{\xi}(u) d u d w \\
= & \frac{1}{h} \int_{\mathbb{R}^{2}}\left\{\int_{\mathbb{R}^{2}} \frac{1}{2 \pi} e^{-i w(x-u) / h} \phi_{K}(w) d w\right\}(m(u)-m(x)) p_{\xi}(u) d u
\end{aligned}
$$


The expression in curly bracket is by Fourier inversion equal to $K((x-u) / h)$, thus we have proved the first part of (i).

A Taylor-expansion of $m$ and $p_{\xi}$ up to second (first) order yields because of $\int_{\mathbb{R}} v K(v) d v=0:$

$$
\begin{aligned}
& \int_{\mathbb{R}}\{m(u)-m(x)\} \frac{1}{h} K\left(\frac{x-u}{h}\right) p_{\xi}(u) d u \\
= & \int_{\mathbb{R}} K(v)\left\{-h v m^{\prime}(x)+\frac{1}{2} h^{2} v^{2} m^{\prime \prime}\left(\hat{x}_{1}\right)\right\}\left\{p_{\xi}(x)-h v p_{\xi}^{\prime}\left(\hat{x}_{2}\right)\right\} d v \\
= & O\left(h^{2}\right) .
\end{aligned}
$$

$\hat{x}_{1}$ and $\hat{x}_{2}$ denote suitable values between $x-h v$ and $x$, possibly depending on $v$.

(ii) Concerning the variance we obtain

$$
\begin{aligned}
& \operatorname{var}\left(\frac{1}{T h} \sum_{t} K_{h}\left(\frac{x-X_{t}}{h}\right)\left(X_{t+1}-m(x)\right)\right) \\
= & \frac{1}{T h^{2}} \cdot \operatorname{var}\left(K_{h}\left(\frac{x-X_{1}}{h}\right)\left(X_{2}-m(x)\right)\right)+ \\
& +\frac{2}{T^{2} h^{2}} \cdot \sum_{s<t} \operatorname{cov}\left(K_{h}\left(\frac{x-X_{s}}{h}\right)\left(X_{s+1}-m(x)\right), K_{h}\left(\frac{x-X_{t}}{h}\right)\left(X_{t+1}-m(x)\right)\right) .
\end{aligned}
$$

Using a covariance-inequality for strongly mixing sequences with geometrically decaying mixing coefficient (cf. Bosq (1996), Corollary 1.1 (page 19) we obtain the following bound of the above expression

$$
\frac{1}{T h^{2}} \sup _{u \in \mathbb{R}}\left|K_{h}(u)\right|^{2} E\left(X_{2}-m(x)\right)^{2}+\frac{O(1)}{T h^{2}}\left(E\left|K_{h}\left(\frac{x-X_{1}}{h}\right)\left(X_{2}-m(x)\right)\right|\right)^{\frac{2}{2+\delta}}
$$

for $\delta>0$ arbitrarily small. Since

$$
\left(E\left|K_{h}\left(\frac{x-X_{1}}{h}\right)\left(X_{2}-m(x)\right)\right|^{2+\delta}\right)^{\frac{2}{2+\delta}} \leq \sup _{u \in \mathbb{R}}\left|K_{h}(u)\right|^{2} \cdot\left(E\left|X_{2}-m(x)\right|^{2+\delta}\right)^{\frac{2}{2+\delta}}
$$

and since, from Fan and Truong (1993), (7.8), we have for $\chi=\frac{1}{4} M_{0} \pi>0$

$$
\sup _{u \in \mathbb{R}}\left|K_{h}(u)\right|=O(h)+O\left(\frac{\exp (\chi / h)}{h}\right),
$$

we can bound the variance through $O\left(\frac{\exp (2 \chi / h)}{T h^{4}}\right)$. This expression converges to zero for $h=c / \log T$ and $c>2 \chi$. 


\section{Proof of Lemma 3.3:}

(i) We have by independence of $\xi_{0}$ and $\varepsilon_{1}$

$$
\begin{aligned}
& E \frac{1}{h} K_{h}\left(\frac{x-X_{1}}{h}\right) \\
= & \frac{1}{h} E K_{h}\left(\frac{x-\xi_{0}-\varepsilon_{1}}{h}\right) \\
= & \frac{1}{h} \int_{\mathbb{R}^{2}} K_{h}\left(\frac{x-u-v}{h}\right) p_{\xi}(u) p_{\varepsilon}(v) d u d v \\
= & \frac{1}{2 \pi h} \int_{\mathbb{R}^{2}} e^{-i \frac{w}{h}(x-u)} \phi_{K}(w) p_{\xi}(u) d u d w \\
= & \frac{1}{h} \int_{\mathbb{R}} K\left(\frac{x-u}{h}\right) p_{\xi}(u) d u \\
= & \int_{\mathbb{R}} p_{\xi}(x-h v) K(v) d v=p_{\xi}(x)+O\left(h^{2}\right) .
\end{aligned}
$$

The last equality is based on a second order Taylor-approximation of $p_{\xi}$.

(ii) Along the same lines as in the proof of Lemma 3.2 we obtain the wanted assertion.

\section{Proof of Lemma 3.5:}

$$
\begin{aligned}
& E \frac{1}{h} \tilde{K}_{h}\left(\frac{x-X_{1}}{h}\right)=\frac{1}{h} E \tilde{K}_{h}\left(\frac{x-m\left(\xi_{0}\right)-\varepsilon_{1}-\eta_{1}}{h}\right) \\
= & \frac{1}{2 \pi h} \int_{\mathbb{R}^{4}} e^{-i w \frac{x-r-s-t}{h}} \phi_{K}(w) \frac{\phi_{\eta}(w / h)}{\phi_{\eta+\varepsilon}(w / h)} d P^{m\left(\xi_{0}\right)}(r) d P^{(\varepsilon, \eta)}(s, t) d w \\
= & \frac{1}{2 \pi h} \int_{\mathbb{R}^{2}} e^{-i w \frac{x-r}{h}} \int_{\mathbb{R}^{2}} e^{i w \frac{s+t}{h}} d P^{(\varepsilon, \eta)}(s, t) d P^{m\left(\xi_{0}\right)}(r) \phi_{K}(w) \frac{\phi_{\eta}^{(w / h)}}{\phi_{\eta+\varepsilon}(w / h)} d w \\
= & \frac{1}{2 \pi h} \int_{\mathbb{R}^{2}} e^{-i w \frac{x-r}{h} \phi_{K}(w) \phi_{\eta}(w / h) d w d P^{m\left(\xi_{0}\right)}(r)} \\
= & \frac{1}{h} \int_{\mathbb{R}^{2}} \frac{h}{\sigma_{\eta}} \varphi\left(\frac{\frac{x-r}{h}-u}{\sigma_{\eta} / h}\right) K(u) d u d P^{m\left(\xi_{0}\right)}(r)
\end{aligned}
$$

because $\phi_{K}(w) \cdot \phi_{\eta}(w / h)$ is the characteristic function of $K * \mathcal{N}\left(0, \sigma_{\eta}^{2} / h^{2}\right)$ with density $\frac{h}{\sigma_{\eta}} \int_{\mathbb{R}} \varphi\left(\frac{-u}{\sigma_{\eta} / h}\right) K(u) d u$. ( $\varphi$ denotes the density of the standard normal distribution) 
and the Fourier inversion formula.

$$
\begin{aligned}
= & \int_{\mathbb{R}}\left\{\frac{1}{\sigma_{\eta}} \int_{\mathbb{R}} \varphi\left(\frac{x-h u-r}{\sigma_{\eta}}\right) d P^{m\left(\xi_{0}\right)}(r)\right\} K(u) d u \\
& \text { The term in curly brackets is the density of } \mathcal{L}\left(m\left(\xi_{0}\right)+\eta_{1}\right) \text { which is } p_{\xi} \\
= & \int_{\mathbb{R}} p_{\xi}(x-h u) K(u) d u=p_{\xi}(x)+O\left(h^{2}\right) \\
& \text { using the usual arguments and } \int_{\mathbb{R}} u K(u) d u=0 .
\end{aligned}
$$

\section{References}

[1] Bosq, D. (1996). Nonparametric Statistics for Stochastic Processes. Lecture Notes in Statistics 110, Springer-Verlag, New York.

[2] Carroll, R.J. and Hall, P. (1988). Optimal rates of convergence for deconvoluting a density. J. Amer. Statist. Assoc. 83, 1184-1186.

[3] Chesney, M. and Scott, L.O. (1989). Pricing European Currency Options: A Comparison of the Modified Black-Scholes Model and a Random Variance Model. J. Financial Quant. Anal. 24, 267-284.

[4] Doukhan, P. (1994). Mixing: Properties on Examples. Lecture Notes in Statistics 85, Springer-Verlag, New York.

[5] Fan, J. (1991a). On the optimal rates of convergence for nonparametric deconvolution problems. Ann. Statist. 19, 1257-1272.

[6] Fan, J (1991b). Asymptotic normality for deconvolution kernel density estimators. Sankhyā Ser. A 53, 97-110.

[7] Fan, J. and Truong, Y.K. (1993). Nonparametric regression with errors in variables. Ann. Statist. 21, 1900-1925.

[8] Gradstein, I. and Ryshik, I. (1981). Tables. Verlag MiR, Moskau and Verlag Harri Deutsch, Thun.

[9] Liu, M.C. and Taylor, R.L. (1989). A consistent nonparametric density estimator for the deconvolution problem. Canad. J. Statist. 17, 427-438.

[10] Scott, L.O. (1987). Option Pricing When the Variance Changes Randomly: Theory, Estimation and an Application. J. Financial Quant. Anal. 22, 419-438.

[11] Scott, L.O. (1991). Random Variance Option Pricing: Empirical Tests of the Model and Delta-Sigma Hedging. Adv. Futures Options Res. 5, 113-135. 
[12] Stefanski, L.A. and Carroll, R.J. (1990). Deconvoluting kernel density estimators. Statistics 21, 169-184.

[13] Taylor, S.J. (1994). Modelling Stochastic Volatility: A Review and Comparative Study. Mathematical Finance 4, 183-204.

[14] Wiggins, J.B. (1987). Option Values Under Stochastic Volatility: Theory and Empirical Estimates. J. Financial Econ. 19, 351-372. 